\title{
Theoretical Analysis of Repetition Time-Switched Transmit Diversity Scheme
}

\author{
Pei Xiao ${ }^{\dagger}$, Boon Kien $\mathrm{Khoo}^{\dagger \dagger}$, Stéphane Y. Le Goff ${ }^{\dagger \dagger}$, Rahim Tafazolli ${ }^{\dagger}$ \\ ${ }^{\dagger}$ Centre for Communication Systems Research (CCSR) \\ University of Surrey, Guildford, UK. E-mail: \{p.xiao,r.tafazolli\}@ surrey.ac.uk \\ ${ }^{\dagger \dagger}$ Cambridge Broadband Networks \\ Cambridge Business Park, Cambridge, UK. E-mail: BKienKhoo@cbnl.com \\ ${ }^{\dagger \dagger}$ School of Electrical, Electronic and Computer Engineering \\ Newcastle University, UK. E-mail: stephane.le-goff@ncl.ac.uk
}

\begin{abstract}
The conventional transmit diversity schemes, such as Alamouti scheme, use several radio frequency (RF) chains to transmit signals simultaneously from multiple antennas. In this paper, we propose a low-complexity repetition time-switched (RTSTD) transmit diversity algorithm, which employs only one RF chain as well as a low-complexity switch for transmission. A mathematical model is developed to assess the performance of the proposed scheme. In order to make it applicable for practical applications, we also investigate its joint application with orthogonal frequency division multiplexing (OFDM) and channel coding techniques to combat frequency selective fading.
\end{abstract}

\section{INTRODUCTION}

Space-time coding (STC) technique, which is categorized by space-time trellis coding (STTC) [1] and space-time block coding (STBC) [2], [3], is a promising approach to achieve high data rate as well as high quality of services requirements. In addition to STC, antenna selection scheme has been introduced as a low-complexity and low-cost approach for multiple-antenna systems [4]. Its main drawback is the requirement of the feedback information from receiver to transmitter. Another simple transmit diversity scheme is to cycle the data stream through the transmit antenna in a round ribbon fashion such that only one antenna is used at a time. This is called time-switched transmit diversity (TSTD) system. Unlike the STC technique, the diversity gain of TSTD scheme is only exploitable with channel coding which takes advantage of the time selectivity [5].

In this paper, we propose a low-complexity repetition timeswitched transmit diversity (RTSTD) scheme for MIMO systems with two transmit antennas. This technique consists of using one RF chain and a low-complexity switch to select antenna sequentially for transmission. A mathematical model is developed to assess the performance of the RTSTD. Then, we discuss its application in association with OFDM and channel coding to combat frequency selective fading and provide diversities in space, time and frequency domains.

Notations: $(\cdot)^{\mathcal{H}}$ denotes matrix/vector conjugate transpose, $(\cdot)^{T}$ denotes matrix/vector transpose, $(\cdot)^{*}$ denotes matrix/vector and complex scalar conjugate, and $\mathrm{E}[\cdot]$ denotes the expectation operation. Lower case bold letters, e.g., $\mathbf{x}$, denote vectors; upper case bold letters, e.g., $\mathbf{X}$, denote matrices.

\section{TRANSMit Diversity SCHEMES}

\section{A. Proposed RTSTD Scheme}

In the proposed RTSTD system, signals are transmitted sequentially from two transmit antennas using one RF chain and a low-complexity switch. At a given time $t$, antenna 1 is selected to transmit signal $x$. Then, at time $t+T$, antenna 2 is selected and the same signal $x$ is transmitted again.

In the receiver, a buffer is employed in order to store the signal transmitted from antenna 1 before combining with the signal transmitted from antenna 2 . The two received signals are given as follows: $y_{1}=h_{1} x+n_{1}, y_{2}=h_{2} x+n_{2}$, where $y_{1}$ and $y_{2}$ are the received signals from antenna 1 and 2 , respectively. Once both signals from antenna 1 and antenna 2 are received, the maximal-ratio combining scheme is then applied to combine both received signals. Hence, the combined signal is given by

$$
z=\left(h_{1}^{*} y_{1}+h_{2}^{*} y_{2}\right) /\left(\left|h_{1}^{2}\right|+\left|h_{2}^{2}\right|\right)
$$

and finally sent to a maximum-likelihood detector. Note that the proposed system employs two transmit antennas and one receive antenna. It is, however, applicable to any configuration with two transmit antennas and an arbitrary number of $n_{R}$ receive antennas.

Considering the fact that the RTSTD system uses only one RF chain and a low-complexity switch, a significant reduction in both complexity and size of the wireless devices can be achieved compared to the conventional space-time coded systems.

Next we conduct a theoretical analysis to derive the bit error performance of the proposed RTSTD system. The decision statistic for the symbol $x$ in (1) can be simplified to

$$
z=x+w,
$$

where $w \sim \mathcal{C} \mathcal{N}\left(0, N_{w}\right), N_{w}=N_{0} / \mathcal{P}$, and $\mathcal{P}=\sum_{i=1}^{L}\left|h_{i}\right|^{2}$.

The BER expression of an $M$-ary square QAM modulated system can be expressed according to [6] as

$$
\begin{aligned}
P_{M}(\mathcal{P}) & =\frac{2(\sqrt{M}-1)}{\sqrt{M} \log _{2} \sqrt{M}} Q\left(\sqrt{\frac{3 \log _{2} M E_{b} \mathcal{P}}{(M-1) N_{0}}}\right) \\
& +\frac{2(\sqrt{M}-2)}{\sqrt{M} \log _{2} \sqrt{M}} Q\left(\sqrt{\frac{27 \log _{2} M E_{b} \mathcal{P}}{(M-1) N_{0}}}\right),
\end{aligned}
$$


where $Q(x)$ is the complementary Gaussian cumulative distribution function [7] given by

$Q(x)=\int_{x}^{\infty} \frac{1}{\sqrt{2 \pi}} \exp \left(-t^{2} / 2\right) d t=P_{r}\{t>x\}=P_{r}\{t<-x\}$

In order to obtain the average bit error probability for the RTSTD scheme, we need to average $P_{M}(\mathcal{P})$ over the distribution of the random variable $\mathcal{P}$, i.e.,

$$
\begin{aligned}
P_{b} & =\int_{0}^{\infty} P_{M}(\mathcal{P}) f(\mathcal{P}) d \mathcal{P} \\
& =\frac{2(\sqrt{M}-1)}{\sqrt{M} \log _{2} \sqrt{M}} \int_{0}^{\infty} Q\left(\sqrt{\frac{3 \log _{2} M E_{b} \mathcal{P}}{(M-1) N_{0}}}\right) f(\mathcal{P}) d \mathcal{P} \\
& +\frac{2(\sqrt{M}-2)}{\sqrt{M} \log _{2} \sqrt{M}} \int_{0}^{\infty} Q\left(\sqrt{\frac{27 \log _{2} M E_{b} \mathcal{P}}{(M-1) N_{0}}}\right) f(\mathcal{P}) d \mathcal{P}
\end{aligned}
$$

where $f(\mathcal{P})$ is the probability density function (PDF) of $\mathcal{P}$.

In real propagation environments, MIMO channels are correlated to a certain extend due to limited antenna element separation, geometry of array and surrounding objects in the near field of antenna elements, etc. [8]. Antenna correlation is defined as the envelope correlation coefficient between signals received at an antenna element, i.e.,

$$
\begin{aligned}
\rho & =\left|\frac{\mathrm{E}\left\{\left(h_{i}-E\left\{h_{i}\right\}\right)\left(h_{m}-E\left\{h_{m}\right\}\right)^{*}\right\}}{\sqrt{\mathrm{E}\left\{\left|h_{i}-E\left\{h_{i}\right\}\right|^{2}\right\} \mathrm{E}\left\{\left|h_{m}-E\left\{h_{m}\right\}\right|^{2}\right\}}}\right| \\
& =\left|\frac{\operatorname{cov}\left(h_{i}, h_{m}\right)}{\sqrt{\operatorname{var}\left(h_{i}\right) \operatorname{var}\left(h_{m}\right)}}\right|,
\end{aligned}
$$

Finding $f(\mathcal{P})$ (the probability density function (PDF) of $\mathcal{P}$ in Eq. (5)) is tedious in the presence of antenna correlation. To work around this problem, we form a channel vector $\mathbf{h}=\left[\begin{array}{llll}h_{1} & h_{2} & \ldots & h_{L}\end{array}\right]^{T}$. The joint PDF of $\mathbf{h}$ is determined by its mean vector $\mathbf{m}_{\mathbf{h}}$ and its covariance matrix $\mathbf{R}=$ $\mathrm{E}\left\{\left[\mathbf{h}-\mathbf{m}_{\mathbf{h}}\right]\left[\mathbf{h}-\mathbf{m}_{\mathbf{h}}\right]^{\mathcal{H}}\right\}$, i.e.,

$p(\mathbf{h})=\frac{1}{(2 \pi)^{2}(\operatorname{det} \mathbf{R})^{1 / 2}} \exp \left[-\frac{1}{2}\left(\mathbf{h}-\mathbf{m}_{\mathbf{h}}\right)^{\mathcal{H}} \mathbf{R}^{-1}\left(\mathbf{h}-\mathbf{m}_{\mathbf{h}}\right)\right]$.

Note that the antenna correlation is taken into account in the covariance matrix $\mathbf{R}$. Since $\mathcal{P}=\mathbf{h}^{\mathcal{H}} \mathbf{h}$, in the presence of spatial correlation, Eq. (5) can be reformed as

$$
\begin{aligned}
P_{b} & =\int_{0}^{\infty} P_{b}(\mathcal{P}) f(\mathcal{P}) d \mathcal{P} \\
& =\frac{2(\sqrt{M}-1)}{\sqrt{M} \log _{2} \sqrt{M}} \int_{\mathbf{h}} Q\left(\sqrt{\frac{3 \log _{2} M E_{b} \mathbf{h}^{\mathcal{H}} \mathbf{h}}{(M-1) N_{0}}}\right) f(\mathbf{h}) d \mathbf{h} \\
& +\frac{2(\sqrt{M}-2)}{\sqrt{M} \log _{2} \sqrt{M}} \int_{\mathbf{h}} Q\left(\sqrt{\frac{27 \log _{2} M E_{b} \mathbf{h}^{\mathcal{H}} \mathbf{h}}{(M-1) N_{0}}}\right) f(\mathbf{h}) d \mathbf{h} .
\end{aligned}
$$

The conventional definition of the Q-function is given by $Q(x)=\int_{x}^{\infty} \frac{1}{\sqrt{2 \pi}} \exp \left(-\frac{y^{2}}{2}\right) d y$, which is substituted into (8) to derive the average bit error probability. However, the analysis is difficult to perform in this way since the argument $x$ appears in the lower limit of the integral. The problem can be tackled by using the alternative representation of the Gaussian $Q$-function [9]

$$
Q(x)=\frac{1}{\pi} \int_{0}^{\pi / 2} \exp \left(-\frac{x^{2}}{2 \sin ^{2} \theta}\right) d \theta .
$$

Substituting (9) into (8), the average bit error probability for the RTSTD scheme can be derived according to [10] as

$$
\begin{aligned}
P_{b} & =\frac{2(\sqrt{M}-1)}{\sqrt{M} \log _{2} \sqrt{M} \pi} \int_{0}^{\frac{\pi}{2}}\left[\operatorname{det}\left(\frac{\boldsymbol{\Gamma}_{1}}{\sin ^{2} \theta}+\mathbf{I}\right)\right]^{-1} d \theta \\
& +\frac{2(\sqrt{M}-2)}{\sqrt{M} \log _{2} \sqrt{M} \pi} \int_{0}^{\frac{\pi}{2}}\left[\operatorname{det}\left(\frac{\boldsymbol{\Gamma}_{2}}{\sin ^{2} \theta}+\mathbf{I}\right)\right]^{-1} d \theta,
\end{aligned}
$$

where $\boldsymbol{\Gamma}_{1}=\mathbf{R} / \beta_{1}^{2}, \boldsymbol{\Gamma}_{2}=\mathbf{R} / \beta_{2}^{2}$, and

$$
\beta_{1}=\sqrt{\frac{2(M-1) N_{0}}{3 \log _{2} M E_{b}}}, \quad \beta_{2}=\sqrt{\frac{2(M-1) N_{0}}{27 \log _{2} M E_{b}}} .
$$

Eq. (10) is derived for the general case with spatial correlation. In the sequel, we derive the performance in a special case when spatial correlation is absent. To this end, we need to find a closed-form solution for the integral

$$
P_{a}=\frac{1}{\pi} \int_{0}^{\frac{\pi}{2}}\left[\operatorname{det}\left(\frac{\boldsymbol{\Sigma}}{\sin ^{2} \theta}+\mathbf{I}\right)\right]^{-1} d \theta
$$

where $\boldsymbol{\Sigma}$ stands for either $\boldsymbol{\Gamma}_{1}$ or $\boldsymbol{\Gamma}_{2}$. In this case, $\boldsymbol{\Sigma}=\mathbf{R} / \beta^{2}=$ $c \mathbf{I}$, where $\beta$ stands for either $\beta_{1}$ or $\beta_{2}$, and $c$ stands for either $c_{1}=\gamma / \beta_{1}^{2}$ or $c_{2}=\gamma / \beta_{2}^{2}$. To simplify analysis, we assume $\gamma_{i}=\mathrm{E}\left[\left|h_{i}\right|^{2}\right]=\gamma, \quad i=1, \ldots, L\left(L=2 n_{R}\right)$.

Denoting $\psi(\theta)=\frac{c}{\sin ^{2} \theta}+1$, and substituting it to (11) yields

$$
\begin{aligned}
P_{a} & =\frac{1}{\pi} \int_{0}^{\frac{\pi}{2}}\left[\operatorname{det}\left(\frac{\boldsymbol{\Sigma}}{\sin ^{2} \theta}+\mathbf{I}\right)\right]^{-1} d \theta \\
& =\frac{1}{\pi} \int_{0}^{\frac{\pi}{2}}\left(\frac{c}{\sin ^{2} \theta}+1\right)^{-L} d \theta \\
& =\frac{1}{\pi} \int_{0}^{\frac{\pi}{2}} \psi(\theta)^{-L} d \theta=\frac{1}{\pi \Gamma(L)} \int_{0}^{\frac{\pi}{2}} \frac{\Gamma(L)}{\psi(\theta)^{L}} d \theta
\end{aligned}
$$

where the Gamma function is defined according to [11, p. 884] as

$$
\Gamma(L)=x^{L} \int_{0}^{\infty} e^{-x t} t^{L-1} d t, \quad x, L>0 .
$$

Substituting (13) into (12), we obtain

$$
\begin{aligned}
P_{a} & =\frac{1}{\pi \Gamma(L)} \int_{0}^{\frac{\pi}{2}} \frac{\psi(\theta)^{L}}{\psi(\theta)^{L}} \int_{0}^{\infty} e^{-\psi(\theta) t} t^{L-1} d t d \theta \\
& =\frac{1}{\Gamma(L)} \int_{0}^{\infty}\left(\frac{1}{\pi} \int_{0}^{\frac{\pi}{2}} e^{-c t / \sin ^{2} \theta} d \theta\right) e^{-t} t^{L-1} d t .
\end{aligned}
$$

Based on the alternative definition of the Q-function in (9), the inner integral in (14) can be expressed as

$$
\frac{1}{\pi} \int_{0}^{\frac{\pi}{2}} e^{-c t / \sin ^{2} \theta} d \theta=\frac{1}{\pi} \int_{0}^{\frac{\pi}{2}} e^{\frac{-(\sqrt{2 c t})^{2}}{2 \sin ^{2} \theta}} d \theta=Q(\sqrt{2 c t}) .
$$


Substituting (15) into (14) yields

$$
\begin{aligned}
P_{a} & =\frac{1}{\Gamma(L)} \int_{0}^{\infty} Q(\sqrt{2 c t}) e^{-t} t^{L-1} d t \\
& =\frac{1}{\Gamma(L) \gamma^{L}} \int_{0}^{\infty} Q\left(\sqrt{\frac{2 c \mathcal{P}}{\gamma}}\right) e^{-\mathcal{P} / \gamma} \mathcal{P}^{L-1} d \mathcal{P} \\
& =\frac{1}{\gamma^{L}(L-1) !} \underbrace{\int_{0}^{\infty} Q\left(\sqrt{\frac{2 c \mathcal{P}}{\gamma}}\right) \mathcal{P}^{L-1} \exp (-\mathcal{P} / \gamma) d \mathcal{P}}_{\Phi} .
\end{aligned}
$$

The second equality in (16) is derived by assigning $t=$ $\mathcal{P} / \gamma$. The third equality in (16) holds since $\Gamma(L)=(L-1)$ ! [7] for positive integer $L$. The integral in (16) can be solved using the following equation [12]

$$
\begin{aligned}
& \int_{0}^{\infty} Q(\sqrt{2 a x}) x^{L-1} \exp (-x / \gamma) d x \\
& =\frac{1}{2} \gamma^{L}(L-1) !\left[1-\sum_{k=0}^{L-1} \nu\left(\frac{1-\nu^{2}}{4}\right)^{k}\left(\begin{array}{c}
2 k \\
k
\end{array}\right)\right],
\end{aligned}
$$

where $\nu=\sqrt{a \gamma /(2+a \gamma)}$. Assigning $a=2 c / \gamma$, we obtain the integral in (16) as

$$
\Phi=\frac{1}{2} \gamma^{L}(L-1) !\left[1-\sum_{k=0}^{L-1} \mu\left(\frac{1-\mu^{2}}{4}\right)^{k}\left(\begin{array}{c}
2 k \\
k
\end{array}\right)\right]
$$

where $\mu=\sqrt{\frac{a \gamma}{2+a \gamma}}=\sqrt{\frac{c}{1+c}}$.

Based on the above analysis, we can readily prove that

$$
\begin{aligned}
& \frac{1}{\pi} \int_{0}^{\frac{\pi}{2}}\left[\operatorname{det}\left(\frac{\boldsymbol{\Gamma}_{1}}{\sin ^{2} \theta}+\mathbf{I}\right)\right]^{-1} d \theta \\
& =\frac{1}{2}\left[1-\sum_{k=0}^{L-1} \mu_{1}\left(\frac{1-\mu_{1}^{2}}{4}\right)^{k}\left(\begin{array}{c}
2 k \\
k
\end{array}\right)\right] ; \\
& \frac{1}{\pi} \int_{0}^{\frac{\pi}{2}}\left[\operatorname{det}\left(\frac{\boldsymbol{\Gamma}_{2}}{\sin ^{2} \theta}+\mathbf{I}\right)\right]^{-1} d \theta \\
& =\frac{1}{2}\left[1-\sum_{k=0}^{L-1} \mu_{2}\left(\frac{1-\mu_{2}^{2}}{4}\right)^{k}\left(\begin{array}{c}
2 k \\
k
\end{array}\right)\right],
\end{aligned}
$$

where

$$
\begin{array}{rlrl}
\mu_{1} & =\sqrt{\frac{a_{1} \gamma}{2+a_{1} \gamma}} ; & a_{1}=\frac{3 \log _{2} M E_{b}}{(M-1) N_{0}} \\
\mu_{2}=\sqrt{\frac{a_{2} \gamma}{2+a_{2} \gamma}} ; & a_{2}=\frac{27 \log _{2} M E_{b}}{(M-1) N_{0}} .
\end{array}
$$

Therefore, we have the average bit error rate for the RTSTD scheme with general $M$-ary square QAM modulation as

$$
\begin{aligned}
P_{b} & =\frac{(\sqrt{M}-1)}{\sqrt{M} \log _{2} \sqrt{M}}\left[1-\sum_{k=0}^{L-1} \mu_{1}\left(\frac{1-\mu_{1}^{2}}{4}\right)^{k}\left(\begin{array}{c}
2 k \\
k
\end{array}\right)\right] \\
& +\frac{(\sqrt{M}-2)}{\sqrt{M} \log _{2} \sqrt{M}}\left[1-\sum_{k=0}^{L-1} \mu_{2}\left(\frac{1-\mu_{2}^{2}}{4}\right)^{k}\left(\begin{array}{c}
2 k \\
k
\end{array}\right)\right] .
\end{aligned}
$$

\section{B. Proposed OFDM-RTSTD Scheme}

MIMO systems provide an effective means of achieving high data rate transmission without increasing the total transmission power or bandwidth for wireless systems [13]. On the other hand, OFDM [14], [15] is well suited for broadband applications due to its robustness against multipath fading by transforming a frequency selective channel into parallel flat fading channels. For wideband signals and frequency selective channels, the multipath interference can be easily alleviated by combining OFDM with the MIMO structure due to OFDM's capability to effectively combat intersymbol interference (ISI). The combination of MIMO and OFDM thus provides a powerful measure to build up a reliable broadband wireless communication system. In order to exploit diversity in an OFDM system, channel coding has to be applied. In this section, we discuss how the proposed transmit diversity scheme can be applied to the coded MIMO-OFDM systems.

At the transmitter, the information sequence $\left\{b_{n}\right\}$ is encoded generating a sequence $\left\{u_{n}\right\}$ of code bits, which is then interleaved to produce a sequence $\left\{v_{n}\right\}$. The modulator converts groups of $M$ code bits into one of $\log _{2} M$ complex symbols from a unit energy signal constellation. The modulated symbols $\left\{s_{n}\right\}$ are then grouped into blocks of size $N$ to form a symbol vector $\mathbf{s}=\{s(1), s(2), \ldots, s(N)\}$, where $N$ is the number of sub-carriers used in OFDM.

The transmitted sequence at each signaling interval undergoes a serial-to-parallel (S/P) conversion and the output block of $N$ parallel symbols is modulated using an inverse fast Fourier transform (IFFT). Note that both the FFT and IFFT operations can be expressed as $N \times N$ matrices denoted as $\mathbf{Q}$ and $\mathbf{Q}^{-1}$, respectively. The $(k, l)$-th entry of the FFT matrix $\mathrm{Q}$ is

$$
\mathbf{Q}_{k, l}=\frac{1}{\sqrt{N}} e^{-j(2 \pi / N) k l} ; \quad k, l=0, \ldots, N-1 .
$$

Note that $\mathbf{Q}$ is an unitary matrix, therefore $\mathbf{Q}^{-1}=\mathbf{Q}^{\mathcal{H}}$, and $\mathbf{Q Q}^{\mathcal{H}}=\mathbf{Q}^{\mathcal{H}} \mathbf{Q}=\mathbf{I}$, where $\mathbf{I}$ denotes the identity matrix.

The output of the IFFT is a block of symbols $\mathbf{x}=$ $\{x(1), x(2), \ldots, x(N)\}$ given by $\mathbf{x}=\mathbf{Q}^{\mathcal{H}} \mathbf{s}$. A parallel-toserial (P/S) converter multiplexes the $N$ parallel symbols and a cyclic prefix $(\mathrm{CP})$ is inserted with duration longer than the impulse response of the channel to combat intersymbol and intercarrier interference. Finally, the OFDM signal is digital-to-analogue (D/A) converted and transmitted over the channel. At first signaling interval, the modulated OFDM signal is transmitted from the first antenna; at the second signaling interval, the same signal is transmitted from the second antenna.

At the receiver, $n_{R}$ streams are processed by an equal number of OFDM reception chains. In particular, the cyclic prefix of each OFDM signal is removed at each signaling interval. The received signal $\mathbf{r}_{j}$ of length $N$ at the $j$-th OFDM chain can be expressed as $\mathbf{r}_{j}=\mathbf{H}_{j} \mathbf{x}+\mathbf{w}_{j}$, where $\mathbf{H}_{j}=\left[\begin{array}{ll}\mathbf{H}_{j, 1} & \mathbf{H}_{j, 2}\end{array}\right]$. Here, $\mathbf{H}_{j, i}$ is an $N \times N$ circulant matrix that contains the channel impulse response from transmit antenna $i$ to receive antenna $j$, whilst $\mathbf{w}_{j}$ is a column vector of length $N$ whose elements are uncorrelated circularly symmetric complex Gaussian random variables with mean zero and variance $N_{0}$. 
Circulant matrices, such as $\mathbf{H}_{j, i}$, hold the important property of being diagonalizable by the Fourier transformation matrix under the condition that the channel is time-invariant over an OFDM symbol, i.e.,

$$
\mathbf{H}_{j, i}=\mathbf{Q}^{\mathcal{H}} \boldsymbol{\Xi}_{j, i} \mathbf{Q}
$$

where $\boldsymbol{\Xi}_{j, i}$ is an $N \times N$ diagonal matrix whose $n$-th diagonal element $\xi_{n}^{(j, i)}, n=1, \ldots, N$, is the channel frequency response at the $n$-th subcarrier. Expression (21) can be easily extended to $\mathbf{H}_{j}=\mathbf{Q}^{\mathcal{H}} \boldsymbol{\Xi}_{j} \mathbf{Q}$, where $\boldsymbol{\Xi}_{j}=\left[\boldsymbol{\Xi}_{j, 1} \boldsymbol{\Xi}_{j, 2}\right]$. Performing FFT operation on $\mathbf{r}_{j}$, we obtain

$$
\begin{aligned}
\mathbf{z}_{j} & =\mathbf{Q} \mathbf{r}_{j}=\mathbf{Q}\left[\mathbf{H}_{j} \mathbf{x}+\mathbf{w}_{j}\right] \\
& =\mathbf{Q}\left[\left(\mathbf{Q}^{\mathcal{H}} \boldsymbol{\Xi}_{j} \mathbf{Q}\right) \mathbf{Q}^{\mathcal{H}} \mathbf{s}+\mathbf{w}_{j}\right] \\
& =\boldsymbol{\Xi}_{j} \mathbf{s}+\mathbf{v}_{j},
\end{aligned}
$$

The above equation holds since $\mathbf{Q}$ is an unitary matrix and $\mathbf{x}=\mathbf{Q}^{\mathcal{H}} \mathbf{s}, \mathbf{v}_{j}=\mathbf{Q} \mathbf{w}_{j}$.

In the case when $n_{R}=1$, the received signal after $\mathrm{CP}$ removal and FFT operation can be written as

$$
\mathbf{z}_{1}=\boldsymbol{\Xi}_{1,1} \mathbf{s}+\mathbf{v}_{1} ; \quad \mathbf{z}_{2}=\boldsymbol{\Xi}_{1,2} \mathbf{s}+\mathbf{v}_{2}
$$

where $\mathbf{z}_{1}$ and $\mathbf{z}_{2}$ are the outputs of the OFDM chain at the first and second signaling interval, respectively. The combined signal is given by

$$
\begin{aligned}
\mathbf{y} & =\boldsymbol{\Xi}_{1,1}^{\mathcal{H}} \mathbf{z}_{1}+\boldsymbol{\Xi}_{1,2}^{\mathcal{H}} \mathbf{z}_{2} \\
& =\left(\left|\boldsymbol{\Xi}_{1,1}\right|^{2}+\left|\boldsymbol{\Xi}_{1,2}\right|^{2}\right) \mathbf{s}+\boldsymbol{\Xi}_{1,1}^{\mathcal{H}} \mathbf{v}_{1}+\boldsymbol{\Xi}_{1,2}^{\mathcal{H}} \mathbf{v}_{2} \\
& =\boldsymbol{\Lambda} \mathbf{s}+\mathbf{w}
\end{aligned}
$$

where $\boldsymbol{\Lambda}=\left|\boldsymbol{\Xi}_{1,1}\right|^{2}+\left|\boldsymbol{\Xi}_{1,2}\right|^{2}$ is an $N \times N$ diagonal matrix whose $(n, n)$-th element $\lambda_{n}=\left|\xi_{n}^{(1,1)}\right|^{2}+\left|\xi_{n}^{(1,2)}\right|^{2}$. The vector $\mathbf{w}=\boldsymbol{\Xi}_{1,1}^{\mathcal{H}} \mathbf{v}_{1}+\boldsymbol{\Xi}_{1,2}^{\mathcal{H}} \mathbf{v}_{2}$ represents the combined noise. Consequently, a set of $N$ parallel channels have been created, and each element of the signal vector $\mathbf{s}$ can be decoded independently.

\section{Numerical and Analytical Results}

The simulated MIMO system has 2 transmit antennas and 1 to 4 receive antennas. The transmit power is set to $n_{T} \sigma_{s}^{2}=2$, i.e., unit average transmit power is used for each transmitted symbol. We first assume uncorrelated Rayleigh fading channel and the channel matrix is normalized such that the average channel gain for each transmitted symbol is equal to unity. In this case, the value of $\gamma$ in Eqs. (19) and (20) is $\gamma=1 / n_{R}$.

In Fig. 1, we compare the simulation results with the analytical results which are obtained by evaluating Eq. (20) for the proposed RTSTD scheme. Close agreement between the simulation results and analytical results for all the antenna setups $\left(n_{R}=1,2,3,4\right)$ have been observed from the figures. This verifies the accuracy of our theoretical analyses, which provide us an insight into the behavior of the RTSTD system.

Fig. 2 shows the performance comparison in $2 \times 1$ and $2 \times 2$ MIMO RTSTD systems with different levels of spatial correlations $(\rho=0.0,0.4,0.8)$. The results are derived by numerically evaluating the single finite-range integral in (10). Apparently, antenna correlation has detrimental effect for the system, and the performance becomes worse as the correlation coefficient $\rho$ increases. By comparing two curves corresponding to $\rho=0.0$ and $\rho=0.4$, one can come to the conclusion that moderate correlation can be tolerated without causing significant performance degradation. Generally speaking, it is desirable to reduce the correlations in the practical MIMO systems, for example, by increasing the antenna spacing at the transmitter and at the receiver.

Fig. 3 shows the performance of the proposed OFDMRTSTD scheme. The number of antennas are chosen to be $n_{T}=n_{R}=2$, the FFT size is 1024 , the length of CP is 64, the power loss incurred by the insertion of $\mathrm{CP}$ is taken into account in the SNR calculation. We use three convolutional codes: the first code has a rate of $1 / 2$, generator polynomial $(5,7)$ and constraint length of 3 ; the second code has a rate of $1 / 2$, generator polynomial $(133,171)$ and constraint length of 7 ; the third code has a rate of $1 / 4$, generator polynomial $(51,55,67,77)$ and constraint length of 6 . The employed modulation scheme is 16-QAM. The soft demapper is designed according to [16]. For the multipath channels, we adopt the SUI-3 channel model [17] used for the 802.16d fixed WiMAX systems [18], the antenna correlation coefficient is set to be $\rho=0.4$. As can be seen from Fig. 3, the performance of the proposed OFDM-RTSTD system improves as the channel code becomes stronger. For comparison purposes, the performance of the uncoded systems is also provided in the figure, where one can see that the channel coding significantly improves the system performance, even the system with a weak $(5,7)$ code performs much better than the uncoded system.

\section{CONCLUSIONS}

A simple RTSTD transmit diversity strategy has been proposed in this paper. It employs only one RF chain and a lowcomplexity switch for transmission, resulting in a significant reduction in complexity and size of the wireless devices. For the uncoded systems, we established a mathematical model to calculate the bit error rate performance of the proposed RTSTD scheme. Our theoretical analysis has been verified by simulation results and proved to be accurate. The proposed RTSTD scheme in its original form does not work for frequency selective channels. We have demonstrated that when applying in conjunction with OFDM and channel coding, the OFDMRTSTD scheme provides an effective solution for practical wireless applications.

\section{REFERENCES}

[1] V. Tarokh, N. Seshadri and A. R. Calderbank. "Space-time codes for high data rate wireless communication: performance criterion and code construction". IEEE Transactions on Information Theory, vol. 44, pp. 744-765, March 1998.

[2] A. Alamouti. "A simple transmit diversity technqiue for wireless communications". IEEE Journal on Selected Areas in Communications, vol. 16, no. 8, pp. 1451-1458, Oct. 1998.

[3] V. Tarokh, H. Jafarkhani and A. R. Calderbank. "Space-time block codes from orthogonal designs". IEEE Transactions on Information Theory, vol. 45, no. 5, pp. 1456-1467, July 1999.

[4] S. Sanayei, A. Nosratinia. "Antenna selection in MIMO systems". IEEE Commun. Mag., vol. 42, no. 10, pp. 68-73, Oct. 2004.

[5] M. Ivrlac and W. Utschick, "On time-switched space-time transmit diversity in MISO systems," IEEE VTC'2002, vol. 2, pp. 710-714, 2002.

[6] K. Cho, D. Yoon. "On the general BER expression of one- and twodimensional amplitude modulation", IEEE Transactions on Communications, vol. 50, no. 7, pp. 1074-1080, July 2002. 


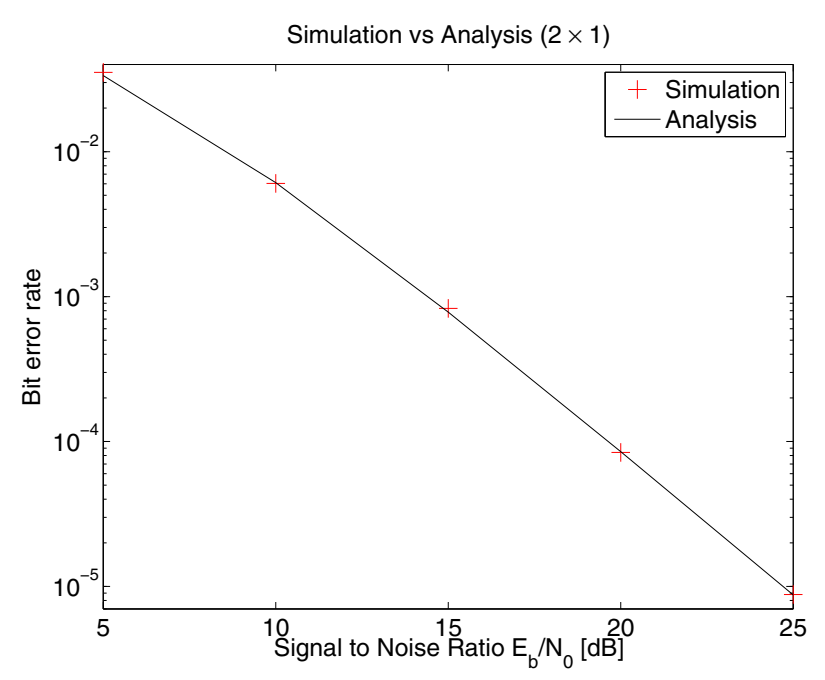

(a) $2 \times 1$ system.

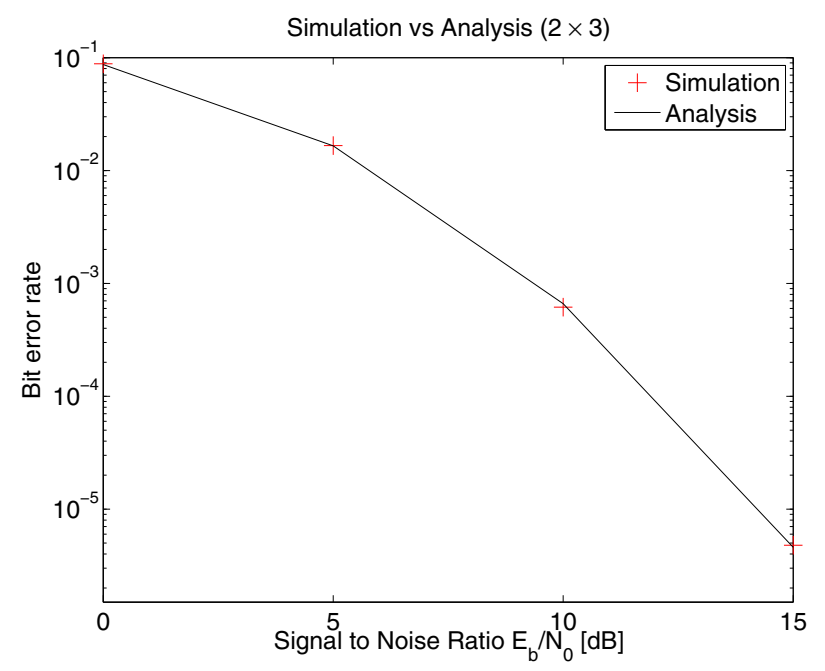

(c) $2 \times 3$ system.

Fig. 1. Comparison between analytical and simulation results.

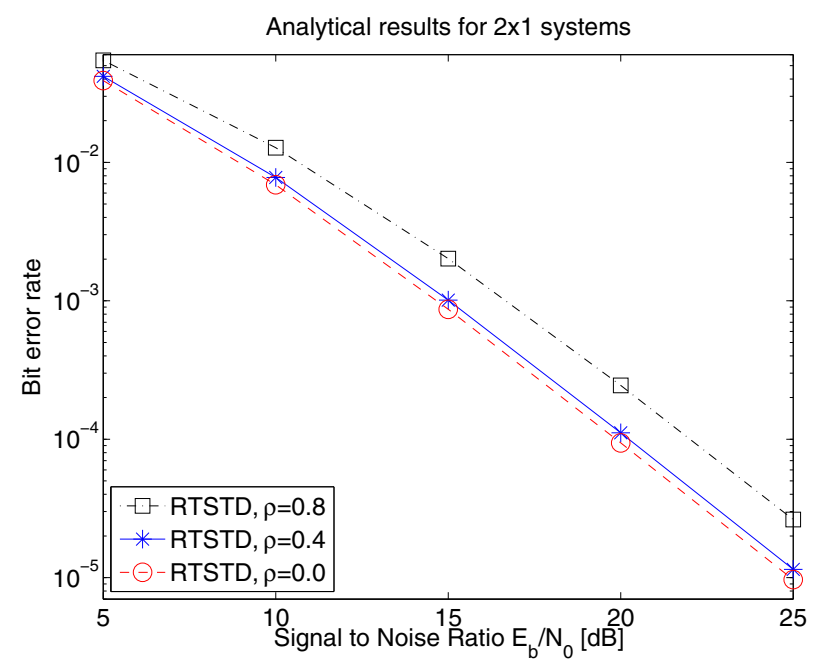

(a) $2 \times 1$ system.

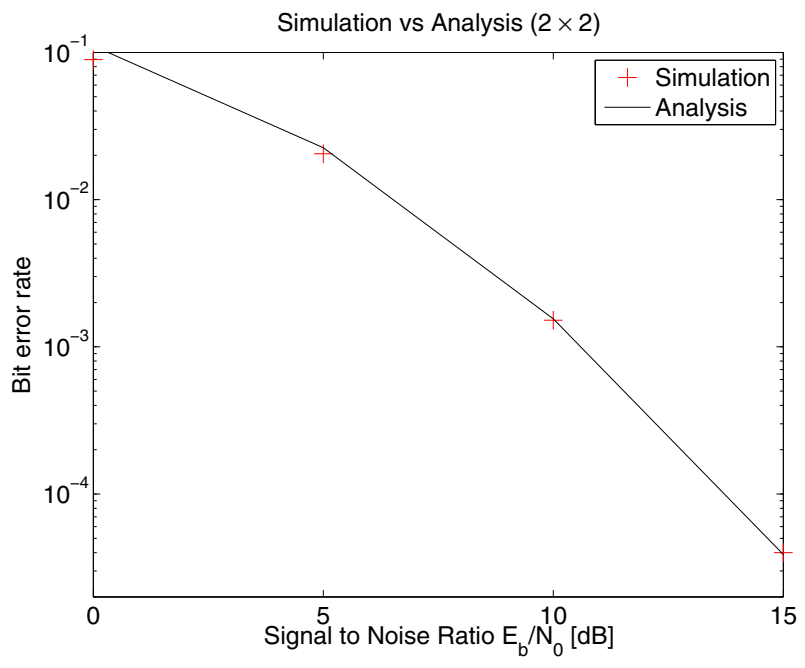

(b) $2 \times 2$ system.

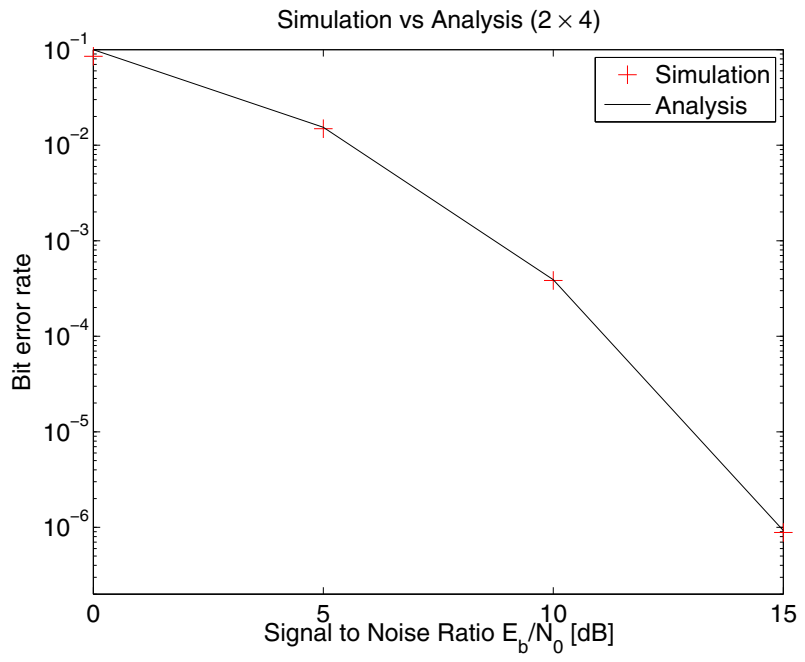

(d) $2 \times 4$ system.

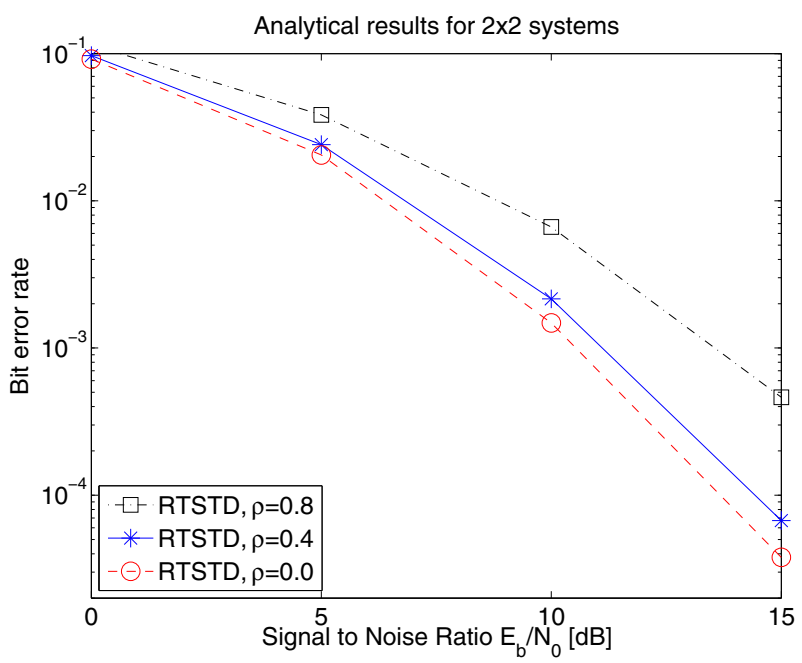

(b) $2 \times 2$ system.

Fig. 2. Performance of RTSTD in presence of antenna correlation. 


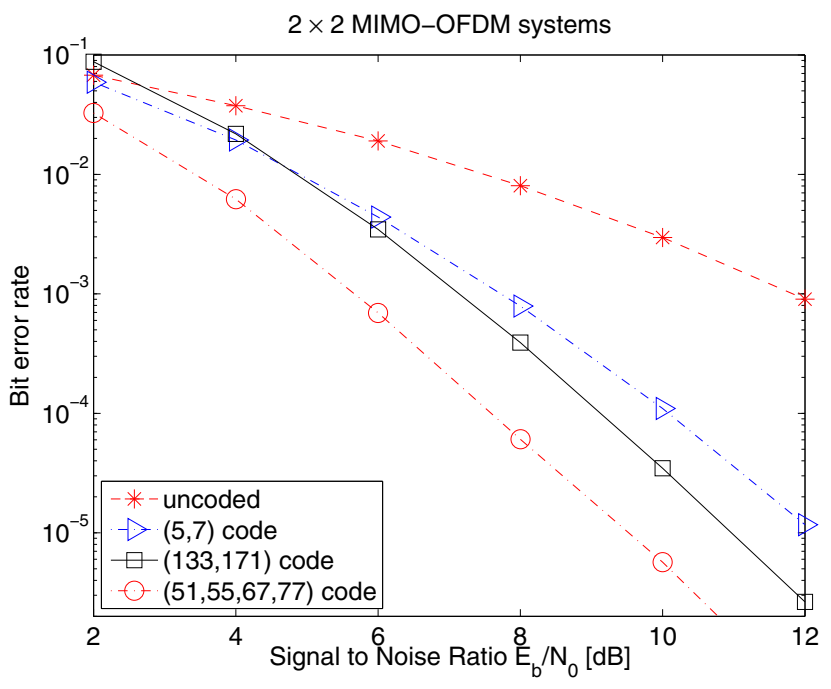

Fig. 3. RTSTD scheme for MIMO-OFDM.

[7] J. Proakis. Digital Communications, 3rd edition, McGraw-Hill, 1995.

[8] M. K. Ozdemir, E. Arvas. "Dynamics of spatial correlation and implications on MIMO systems". IEEE Communications Magazine, vol. 42, no. 6, pp.514-519, June 2004.

[9] M. Simon, M. Alouini. Digital Communication over Fading Channels A Unified Approach to Performance Analysis, John Wiley \& Sons, 2000.

[10] V. Veeravalli. "On performance analysis for signalling on correlated fading channels". IEEE Transactions on Communications, vol. 49, no. 11, pp. 1879-1883, Nov. 2001.

[11] I. Gradshteyn, I. Ryzhik. Table of Integrals, Series, and Products, 6th edition, Academic Press, 2000.

[12] P. Xiao, L. Barbero, M. Sellathurai, T. Ratnarajah. "On the uncoded BER performance bound of the IEEE 802.16d channel". IEEE Signal Processing Letters, vol. 15, pp. 561-564, 2008.

[13] E. Telatar. "Capacity of multi-antenna Gaussian channels". European Transactions on Telecommunications, vol. 10, no. 6, pp. 585-595, Nov. 1999.

[14] L. Cimini. "Analysis and simulation of a digital mobile channel using orthogonal frequency division multiplexing". IEEE Transactions on Communications, vol. 33, no. 7, pp. 665-675, July 1985.

[15] J. Bingham. "Multicarrier modulation for data transmission: an idea whose time has come". IEEE Communications Magazine, vol. 28, no. 5, pp. 5-14, May 1990.

[16] S. Y. Le Goff, A. Glavieux, C. Berrou. "Turbo-codes and high spectral efficiency modulation". Proc. IEEE International Conference on Communications, vol. 2, pp. 645-649, May, 1994.

[17] V. Erceg et al. "Channel models for fixed wireless applications". IEEE 802.16a cont. IEEE 802.16.3c-01/29r4, June 2003.

[18] IEEE 802.16 Working Group on Broadband Wireless Access Standards. Available at http://grouperieee.org/groups/802/16/. 